\title{
Continuum Dislocations and Active Single-slip Assumption in Micro- Mechanical analysis of Matrix Dominated Composite Unit cell
}

\author{
Temesgen Takele Kasa ${ }^{1,2}$ \\ ${ }^{1}$ Department of Mechanical Engineering, Addis Ababa Science and Technology University, Addis Ababa , Ethiopia \\ ${ }^{2}$ Department of Mechanical Engineering, Pukyong National University, Busan, South Korea \\ *e-mail :-ttkh1@pukyong.ac.kr
}

\begin{abstract}
The key purpose of this paper is to propose a mono-slip dependent continuum dislocation method to MDCS (matrix dominated composite structure) analysis. The methodology focused on the dissipation energy theories using CDM (continuum dislocations method) integrated with the kinematics of small strain. The mathematical modeling of CDM comprises active mono-slip system formulations, thermodynamic dislocation analysis (TDA), energy dissipation analysis on the free form, and the progressions of dislocations. Furthermore, the dissipation energy analysis due to dislocation progression could be formulated in zero and non-zero principle by using an energy minimization technique with variational calculus. The numerical analysis performed by Wolfram Mathematica $\odot$, presented in zero and non-zero energy dissipation forms. The outcomes indicate that the formulated approach could be well-qualified to find optimal analysis results for MDC (matrix dominated composite) materials for the mono-slip system. Generally, this approach confirms its ability to investigate MDCS by including inclusions inside the UC.
\end{abstract}

Keywords: Mono-slip; MDCS; CDM; Distortion; active slip; TDA;

\section{INTRODUCTION}

Composites are broadly applied in structural components due to their capabilities of revealing designated in-plane stiffness, bending stiffness, ultimate strength, or thermal expansion coefficient during deformation[1, 2]. In a micro-mechanical approach, irreversible deformation is expressed by dislocation nucleation and motion of dislocations; which means crystallographic irregularities within the periodic crystal framework. For instance, dislocations are nucleated and gathered to decrease the crystal's energy. The preparation of the dislocation is mainly led by the crystal's energy. Plastic flow breaks up into motion of dislocations, which dissipates energy. Thus, all microstructural analyses related to irreversible deformation need to follow the rules of thermodynamics, which can, in turn, be applied to simulate these analyses related to the multifaceted dislocation distribution. Experimental suggestion indicates that dislocations in a strained crystal do not act in a fully random arrangement nor does the deformed crystal exhibit a homogeneous dislocation density throughout its interior [3-5].

In the micromechanics approach, discrete dislocation analysis is sufficient to describe the individual or small numbers of dislocations. This study focuses on a problem to define strains along the dislocation line inside the dislocation core [6]. Similarly, at the macro level, distinctive features of materials are extensively studied by continuum mechanics. Having this concept in mind, the Continuum mechanics approach which is aiming to incorporate the size dependence of dislocation plasticity by extending classical, local continuum descriptions with nonlocal or strain gradient terms 
based on a huge number of dislocation in continuum fashion. Accordingly, there is a huge gap between micro and macro scale, which demands an appropriate meso-model to connect the two extremes named as continuum dislocation formulations. Continuum dislocation formulations include a material length scale and thus permit size effects to be modeled. The scaled length of the material in a deformed body becomes substantial in the mechanics of the material. This research addresses an important concept that shows, when, and how macroscopic overall mechanical properties like strength, hardness, etc., depending on a natural internal length scale related to the characteristic size of the microstructures in the material.

\section{CONTINUUM SLIP FORMULATION}

Here in this section we can specify the side boundary in free form and clamped micro-boundary conditions of the upper and lower part of the region analyzed. In the plastic theories, the gradient of the total displacement field ' $u$ ' represents the Compatible total displacement field. If there is one dislocation loop lying on the slip plane with the Burgers' vector pointing in the slip direction, then the inverse plastic distortion associated with this loop $\left(-\Upsilon_{i j}\right)$ is given by

$$
-\Upsilon_{i j}=b_{i} n_{j} \delta(s)
$$



Fig-1: Diagram of problem formulation for simple shear of an elastic-plastic layer of thickness $\xi$ with one active slip system

Where, $b_{i}$ denotes Burgers' vector, ' $\mathbf{n}_{\mathbf{j}}$ ' is the normal vector and $\delta(s)$ is plane of slip.
This inverse plastic distortion describes the creation of dislocation by cutting the perfect crystal along the surface ' $\mathbf{S}$ ' and shifting the structure under this surface on one Burgers' vector [7]. In general, the sum of the reversible and the irreversible distortion fields gives the total irreversible distortion,

$$
\Upsilon=\Upsilon^{\mathrm{e}}+\Upsilon^{\mathrm{p}}
$$

Where $\Upsilon^{e}$ and $\Upsilon^{p}$ shows the reversible and irreversible distortion components of the material, respectively. Due to the existence of lines of dislocation, both phases are incompatibly represented by the GND (geometrically necessary dislocations) [8]. For a huge amount of loops pass through the slip planes with the average demission among them being considerably smaller than the material characteristic scale size of the standard piece; we go one step further and propose a unique formulation of total plastic distortions $\Upsilon$ created by this slip system is expressed as

$$
\Upsilon_{i j}=\Upsilon(x) s_{i} m_{j}
$$

Where ' $\boldsymbol{m}_{\mathbf{i}}$ ' stands for normal vector indicating towards the direction of slip and ' $\boldsymbol{s}_{\boldsymbol{i}}$ ' denotes slip direction.

\section{Thermodynamic Principles}

To analyze the UCs, we need to use one of the energy principles, named as the principle of Free energy. The GND energy stems custom two facts: the network of dislocation energy is directly proportional to the DOD (density of dislocation) when the dislocation is small, and there exists an SDD (Saturated dislocation density) which characterizes the closest packing of dislocations of equal signs admissible in the unit cell. Assume that the material is isotropic and contains inclusions, for elastically deformed. Analyzing the variational derivative of the yield condition consider the first case, $\varphi<\pi / 4$ (see fig-1).

Simply, the isotropic elastic property of the cell; both the matrix and the reinforcement material are taken to be isotropic[9]. The elastic moduli, $(\Re)$ for the matrix can be written as in the form

$$
\mathfrak{R}=2 G_{12}\left(I^{\prime}-\frac{v}{1-2 v} \mathrm{I} \otimes \mathrm{I}\right)
$$


Where $G_{12}$ is the shear modulus, $v$ is the poison's ratio, and I and I' are the second and fourth-order identity tensors, respectively.

\section{FORMULATIONS}

To simplify the energy functional equation some dimensionless quantities are introduced. Continuum dislocation formulations in a single slip require key equations: a balance and an evolution. Both have a common number of material and geometry parameters. We aim for a formulation that displays the characteristic features of the model-independent from a particular choice of material parameters.

To this end, we pursue a dimensionless analysis of the two frameworks to study how certain combinations of parameters influence the response predicted by this approach. Dimensionless quantities indicated as the height of the unit cell is considered relative to a domain height characterized by $\bar{x}_{2}$ and the length characterized by ' $w$ '. The newly introduced variable varies on the range. By using the newly introduced variables $\bar{x}_{2}$ the energy functional $(0, \bar{\xi})$ formulated as:

$$
\mathrm{E}=\mathrm{b} \rho_{\mathrm{s}} \Pi\left(\mathrm{wLG}_{12}\right)^{-1}, \overline{\mathrm{x}}_{2}=\mathrm{x}_{2} \mathrm{~b} \rho_{\mathrm{s}}, \bar{\xi}=\xi \mathrm{b} \rho_{\mathrm{s}}
$$

Where $\rho_{s}$ saturated dislocation density is the parametric length. Dislocation appears in crystals to reduce their energy. Energy dissipation analysis is more accurate than any other method to describe the plasticity behavior of materials. The displacement in relations with elastic-plastic responses of the materials generally associated with the continuum dislocation accumulation.

The methods will be implemented to calculate the hysteresis energy, triggered by the inclusions that create resistance in the unit cell. If the dislocation motion resistance cannot vanish, the plastic distortion may evolve if and only if the yield condition $\aleph=K$ is fulfilled[7]. Where $\Gamma$ small scale shear strain. And also dislocation-evolution in composite materials is very sensitive to the interaction of reinforcements and matrices in the interface regions. If $|\aleph|<K$ then $\Upsilon$ is frozen, the density of the dislocation assumed to be fixed and the UCs

$$
\left|\begin{array}{l}
-\Upsilon \sin ^{2} 2 \varphi+\kappa \Upsilon \sin ^{2} 2 \varphi-\cos ^{2} 2 \varphi \Upsilon^{a v e} \\
+k \Upsilon^{a v e} \sin ^{2} 2 \varphi+ \\
k \Upsilon_{, 22} \sin ^{2} \varphi\left(b^{2} \rho_{s}^{2}\right)^{-1}+\Gamma \cos 2 \varphi
\end{array}\right|=K / G_{12} \cos 2 \varphi
$$

\section{NUMERICAL EXPRESSION AND DISCUSSION}

It has been seen that in the unit cell of the composite material model; the continuum dislocation is a complicated function of various parameters, such as the dislocation evolution, the material characteristic length $(l)$, and properties of the fiber/particle and the matrix. As a result, it becomes difficult to determine the equilibrium position of dislocations. In this study, only edge dislocations on a one active slip system are considered, taking a plane of slip is in $\mathrm{x}_{2}$-direction and $\mathrm{x}_{1}$-is the slip direction.

When the UCs are subjected to simple shear, there should be a dislocation in the veins of the matrix. During this process the dislocation entangled by the inclusion and the number of dislocation density increases, i.e., it creates a Harding. The shear stress due to dislocation termed as micro shear stress and the shear stress at the hardening point is referred to as critical shear stress. The equilibrium equation from this analysis is obtained as follows

$$
\tau_{c r}-\tau-\tau_{\text {microstress }}=0
$$

This nucleation strength corresponds to a nucleation distance of $l_{\text {nuc }}=125 \mathrm{~b}$. The $\xi / 2$ (height) of the unit cell, is stated based on the material characteristic length $l$ which is assumed to be $l=4000 b$. The influence of inclusion size is analyzed by changing the ratio $\xi / l[10]$.

For material parameters, representative of the $\mathrm{Al}$ matrix is used for all the computational experiments, for which we make the arbitrary choice explained by different researchers[9]. The other material parameters used are isotropic elastic constants of the material, $\mathrm{E}=62.78 \mathrm{Gpa}, \mathrm{v}=0.33$, where $\mathrm{E}$ is Young's modulus and $\mathrm{v}$ is Poisson's ratio. 


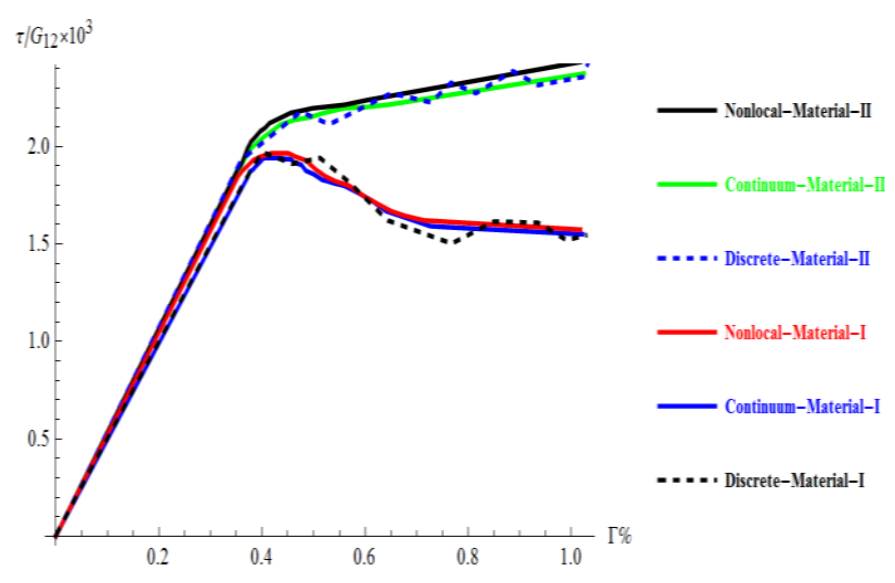

Figure 2- Comparison of $\tau-\Gamma$ for material (I) and (II).

\section{SUMMARY}

In this paper, continuum dislocation theory is discussed to analyze matrix dominated composites in a UCs form. The theory is based on energy characteristics of dislocations combined with typical small-strain continuum dislocation kinematics. The derivations of the continuum dislocation constitute slip formulation, thermodynamic concepts, free energy determinations, threshold value, and evolution of dislocation. Besides, the dissipation and non-dissipation energy forms of the analysis are derived using the variational method and solved by an energy minimization approach. And the outcomes are verified by comparing the existing discrete and non-local dislocation results with the newly formulated continuum dislocation theory results of the same problem.

Furthermore, the effects of material parametric length found in the Continuum dislocation theory analysis are reasonable and consistent with the existing discrete and nonlocal dislocation forecast. Also, this newly formulated theory is fit to the discrete and nonlocal dislocation result of a given similar geometry and size. The capability of the proposed approach is demonstrated by homogenizing composite with a constitute exhibiting various mechanical behaviors.

The proposed method is found to be capable of to handle the elastoplastic phenomenon of composite material and more advanced micromechanics models can be implemented in the proposed approach. Finally, the following conclusions can be drawn, single slip continuum dislocation is modeled, self-stress conditions are involved, two types of material model (Material (i) and Material (ii)) can be incorporated into the proposed approach.

\section{ACKNOWLEDGEMENTS}

I would like to Acknowledge GSM institute for the support of this Research work.

\section{REFERENCES}

[1] T. T. Kasa, "Consideration of interlaminar strainenergy continuity in composite plate analysis using improved higher-order theory," Transactions of the Canadian Society for Mechanical Engineering, vol. 42, pp. 211-221, 2018.

[2] K. Takele, "Interfacial Strain Energy Continuity Assumption-Based Analysis of an Orthotropic-Skin Sandwich Plate Using a Refined Layer-by-Layer Theory," Mechanics of Composite Materials, vol. 54, pp. 281-298, 2018.

[3] D. Weygand, L. Friedman, E. Van der Giessen, and A. Needleman, "Aspects of boundary-value problem solutions with three-dimensional dislocation dynamics," Modelling and Simulation in Materials Science and Engineering, vol. 10, p. 437, 2002.

[4] A. Acharya, "Constitutive analysis of finite deformation field dislocation mechanics," Journal of the Mechanics and Physics of Solids, vol. 52, pp. 301-316, 2004.

[5] D. M. Kochmann, K. Hackl, and K. C. Le, "A continuum-dislocation theory for modeling dislocation microstructures and size effects in crystal plasticity."

[6] E. Kröner, "Continuum theory of defects, in" Physics of defects"(Les Houches, Session 35), ed. R. Balian, et al," ed: North-Holland, Amsterdam, 1980.

[7] V. Berdichevsky and K. Le, "Dislocation nucleation and work hardening in anti-plane constrained shear," Continuum Mechanics and Thermodynamics, vol. 18, pp. 455-467, 2007.

[8] N. Fleck, G. Muller, M. F. Ashby, and J. W. Hutchinson, "Strain gradient plasticity: theory and experiment," Acta Metallurgica et materialia, vol. 42, pp. 475-487, 1994.

[9] A. Acharya and J. Bassani, "Lattice incompatibility and a gradient theory of crystal plasticity," Journal of the Mechanics and Physics of Solids, vol. 48, pp. 1565-1595, 2000.

[10] J. Bassani, A. Needleman, and E. Van der Giessen, "Plastic flow in a composite: a comparison of nonlocal continuum and discrete dislocation predictions," International Journal of Solids and Structures, vol. 38, pp. 833-853, 2001. 